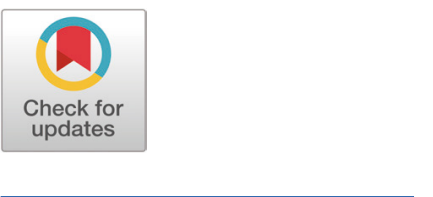

Received: Jan 30, 2020

Revised: Feb 12, 2020

Accepted: Feb 13, 2020

*Corresponding author Jaeyoung Heo

International Agricultural Development and Cooperation Center, Jeonbuk National University, Jeonju 54896, Korea.

Tel: +82-63-270-5926

E-mail: jyheobio@gmail.com

Copyright () 2020 Korean Society of Animal Sciences and Technology. This is an Open Access article distributed under the terms of the Creative Commons Attribution Non-Commercial License (http:// creativecommons.org/licenses/bync/4.0/) which permits unrestricted non-commercial use, distribution, and reproduction in any medium, provided the original work is properly cited.

ORCID

Andrew Wange Bugenyi

https://orcid.org/0000-0001-9036-8391

Ho-Seong Cho

https://orcid.org/0000-0001-7443-167X Jaeyoung Heo

https://orcid.org/0000-0002-9721-8043

Competing interests

No potential conflict of interest relevant to this article was reported.

Funding sources

This research was supported by a grant from the Next-Generation BioGreen 21 Program (PJ01322301, PJ01322302), Rural Development Administration, Korea.

Acknowledgements

The authors thank the management

\section{Association between oropharyngeal microbiome and weight gain in piglets during pre and post weaning life}

\author{
Andrew Wange Bugenyi ${ }^{1}$, Ho-Seong $\mathrm{Cho}^{2}$ and Jaeyoung $\mathrm{Heo}^{3 *}$ \\ ${ }^{1}$ Department of Agricultural Convergence Technology, Jeonbuk National University, Jeonju 54896, Korea \\ ${ }^{2}$ College of Veterinary Medicine and Veterinary Diagnostic Center, Jeonbuk National University, Iksan \\ 54596, Korea \\ ${ }^{3}$ International Agricultural Development and Cooperation Center, Jeonbuk National University, Jeonju \\ 54896, Korea
}

\section{Abstract}

Birth weight and subsequent weight gain is of critical importance in the survival and performance of piglets on a commercial swine farm setting. Oropharyngeal microbiome could influence immunity, and feeding behavior thus impacting health and weight gain. We used $16 \mathrm{~S}$ rRNA gene sequencing to profile the composition and predicted metabolic functionality of the oropharyngeal microbiota in 8 piglets ( 4 with a birthweight $\leq 1.0 \mathrm{~kg}$ and 4 with a birthweight $\geq$ $1.7 \mathrm{~kg}$ ) at 11,26 , and 63 days of age. We found 9 genera that were significantly associated with average daily gain $(A D G)$ at 11 days (false discovery rate, FDR $<0.05$ ) and 26 days of age (FDR $<0.1)$, respectively. The microbial functional profile revealed several pathways associated with ADG (FDR < 0.05). Among these, pathways related to degradation of catechols showed a positive association with ADG at 11,26 , and 63 days of age, implying a potential to breakdown the host-derived catecholamines. We also noted that pathways related to the biodegradation of nucleosides and nucleotides increased with ADG during the pre-weaning phase, while those involved in their biosynthesis decreased. Our findings provide insights into the oropharyngeal microbial memberships and metabolic pathways that are involved in a piglet's weight gain. Thus, providing a basis for the development of strategies aimed at improving weight gain in pigs.

Keywords: Swine, Oropharyngeal, Microbiome, Birthweight, Average daily gain

\section{INTRODUCTION}

Low-birthweight piglets are associated with a reduced survivability and performance [1], thus contributing to the high production costs encountered by many commercial swine enterprises. Most of the management interventions to alleviate these impacts, have been based on optimizing intake of nutritious feed and use of growth promoters, including antibiotics [2]. However, these methods have yielded mixed results. Further, the use of antibiotics could have short- and long-term effects on the health of the animals as well as contribute to the propagation of antimicrobial resistance $[3,4]$.

Meanwhile, recent studies have shown that the microbial composition along the digestive tract has a considerable bearing on the development of the host's immune system [5] and efficiency of nutrient 
and staffs at the Ogeum farm for their permission to use their animals. The authors would also like to thank Joel Bayo and Paul Bogere for their comments which helped to improve this manuscript.

Availability of data and material Upon reasonable request, the datasets of this study can be available from the corresponding author.

Authors' contributions Conceptualization: Bugenyi AW, Heo J. Data curation: Bugenyi AW. Formal analysis: Bugenyi AW, Cho HS. Methodology: Bugenyi AW, Cho HS, Heo J. Software: Bugenyi AW, Cho HS.

Validation: Bugenyi AW, Heo J. Investigation: Bugenyi AW, Heo J. Writing - original draft: Bugenyi AW. Writing - review \& editing: Bugenyi AW, Heo J.

Ethics approval and consent to participate All experimental procedures were approved by the Jeonbuk National University Animal Ethics Committee in accordance with the guidelines of the Korean Council on Animal care (CBNU 2019-037) utilization [6] thus potentially promoting survival and weight gain. Other areas where the microbiota has been found to play important roles include drug metabolism [7] and feeding behavior [8] further affecting health and weight gain, respectively. However, the microbiota is simple and unstable in piglets and has to mature into a relatively more complex and stable community in order to optimally confer these benefits to the animal. Around the time of birth, the neonate receives microbial inocula from its interaction with the sow and the immediate environment [9]. This interaction continues throughout nursing, postweaning and the rest of the pig's life thus shaping the composition of the microbiota. During development, the microbiota can be influenced by several factors including, diet [10], environment [11], weaning [12], antibiotics [13] and probiotics [14].

In order to gain insights into the role played by the microbiota, an understanding of the phylogenetic composition and functional capacity of the microbial community is crucial. With the advent of Next Generation Sequencing techniques, we are able to target and sequence a marker gene such as the $16 \mathrm{~S}$ ribosomal RNA (16S rRNA) gene in order to describe the phylogenetic composition and diversity of the bacteria and archaea within an environment [15]. And by employing a computational model, such as Phylogenetic Investigation of Communities by Reconstruction of Unobserved States (PICRUSt), we are able to reconstruct microbial metagenomes and ultimately predict metabolic pathways within the communities using only this $16 \mathrm{~S}$ rRNA gene sequence data and a reference genomes database [16]. While several studies have been done on the microbial communities in the gastrointestinal tract $[17,18]$, the swine oropharyngeal microbiota remains understudied. Interestingly, the oropharynx is in communication with both the digestive and respiratory tracts which are the major routes of infection by pathogenic organisms in the neonatal and weaning stages of life and thus could greatly influence health and performance.

In the current study, we therefore aimed to profile the bacterial composition and predicted metabolic functions of the oropharyngeal microbial community in low and high birthweight piglets and in piglets with low and high average daily gain (ADG) using next generation sequencing. We hypothesized that the rate of weight-gain (ADG) of the heavier piglets would be associated with a characteristic oropharyngeal microbial signature.

\section{MATERIALS AND METHODS}

\section{Study animals}

The study was conducted on a commercial swine farm with 1,100 breeding sows (Ogeum farm, Yeoju, Korea). A total of 8 mixed sex piglets (3-way crosses; Landrace $\times$ Yorkshire $\times$ Duroc) born on the same day to two 3rd parity sows were used in the study. Four (4) piglets with a birthweight $\leq$ $1.0 \mathrm{~kg}$ and 4 with a birthweight $\geq 1.7 \mathrm{~kg}$ (Table S1), were selected and identified using an oil-based marker that was replenished every week. The body weight of each piglet was measured every week (Table S1), and the average daily gain was calculated by week (Table S2).

The piglets had ad libitum access to water and feed throughout the study. At 10 days of age, they were introduced to a creep feed until weaning after which they were graduated to a post weaning diet (see Table S3 and S4 in the supplementary materials). The commercial feed was based on corn and soybean, however, modifications to the texture and relative composition of additives was made as the piglets grew. Minerals, vitamins, enzymes, probiotics and occasionally antibiotics were added to the feed, based on the particular requirements of the co-housed piglets (see Tables S4 and S5). Prophylactic therapies instituted during this period included; Kanamycin administered intranasally at day 1 to control incidences of atrophic rhinitis; vaccinations against Mycoplasma hyopneumoniae, Porcine circovirus, Erysipellothrix rhusiopatbiae, classical swine fever virus as well as against Foot and Mouth disease virus. In addition, colistin was added into the feed. 


\section{Sample collection}

The oropharyngeal sample was collected by swabbing the soft palate, the root of the tongue and the left and right lateral walls of the oropharyngeal cavity, twice at each surface, using a sterile absorbent swab. The samples were collected at 11,26 , and 63 days of age and stored at $-20^{\circ} \mathrm{C}$ for about 2 weeks before being transferred to the lab where they were kept at $-80^{\circ} \mathrm{C}$.

The piglets were individually weighed weekly and records of clinical condition monitored daily. Coughing, sneezing, diarrhoea, general weakness, fur coat texture, injuries among others were monitored.

\section{DNA extraction}

DNA from the oropharyngeal swab samples was extracted using the Epicenter MasterPure ${ }^{\mathrm{TM}}$ DNA Purification Kit (Epicenter, Madison, WI, USA) following the manufacturer's protocol. First, each sample was rehydrated in $150 \mu \mathrm{L}$ of autoclaved deionized water in a micro-centrifuge tube at room temperature $\left(25^{\circ} \mathrm{C}\right)$ for 30 minutes, vortexed for 15 seconds and then the swab was removed and squeezed against the inside wall of the micro-centrifuge tube. With the resultant solution, the protocol was then followed verbatim. The purity of extracted DNA was assessed by spectrophotometry (NanoDrop Spectrophotometer, Thermo Fisher Scientific, Waltham, MA, USA) through determination of the 260:280 and 260:230 absorbance ratios, while DNA concentration was determined by fluorometry using Qubit HS dsDNA assay kit (Life Technologies, Thermo Fisher Scientific, Waltham, MA, USA). DNA concentration was then normalised to $5 \mathrm{ng} / \mu \mathrm{L}$ and stored at $-20^{\circ} \mathrm{C}$.

\section{Amplification of bacterial $16 \mathrm{~S}$ rRNA gene}

The V4 hypervariable regions of the bacterial $16 \mathrm{~S}$ rRNA gene was amplified using the forward primer, 515F (Parada) (5'-GTGYCAGCMGCCGCGGTAA) [19] and the reverse primer, 806R (Apprill) (5'-GGACTACNVGGGTWTCTAAT) [20]. These primers were designed with illumina overhang adapters that are complementary to illumina sequencing primers. The forward overhang nucleotide sequence (5'-TCGTCGGCAGCGTCAGATGTGTATAAGAGACAG-3') was added to the $515 \mathrm{~F}$ primer while the reverse overhang nucleotide sequence (5'-GTCTCGTGGGCTCGGAGATGTGTATAAGAGACAG-3') was added to the 806R reverse primer. A 25 $\mu \mathrm{L}$ PCR reaction mixture was set up, consisting of $12.5 \mathrm{ng}$ of the DNA template, 1.0 Units of Taq DNA polymerase, $1 \mu \mathrm{M}(5 \mu \mathrm{L})$ of each forward and reverse primers, $200 \mu \mathrm{M}$ of each deoxynucleotide triphosphate (dNTP) and $1 \mathrm{x}$ buffer. Along with the PCR reaction, water as a negative control, a known positive control and extraction control reactions were included. The thermocycler was set at an initial denaturation temperature of $95^{\circ} \mathrm{C}$ for 3 minutes followed by 25 cycles of $95^{\circ} \mathrm{C}$ for 30 seconds, annealing at $56^{\circ} \mathrm{C}$ for 30 seconds and elongation at $72{ }^{\circ} \mathrm{C}$ for 30 seconds followed by a final elongation for 5 minutes.

The amplicon libraries were cleaned up using Agencourt AMPure XP beads (Beckman Coulter, Brea, CA, USA) according to the manufacturer's protocol and then quantified by fluorometry using Qubit High Sensitivity dsDNA assay kit (Life Technologies, Thermo Fisher Scientific, Waltham, MA, USA).

\section{Genomic DNA library preparation and sequencing}

The PCR amplicons were prepared for sequencing by adding indices and illumina sequencing adapters using the Nextera XT DNA index kit (Illumina, San Diego, CA, USA) according to the manufacturer's instructions for single end sequencing. The indexed libraries were then purified using Agencourt AMPure XP beads, quality checked on a Bioanalyzer DNA 1000 chip and then 
quantified by fluorometry using Qubit HS dsDNA assay kit. The libraries were then diluted to an equimolar concentration of $4 \mathrm{nM}$ before pooling for sequencing. A final confirmation of the pooled library concentration was done by a fluorometric measurement before denaturing and sequencing. The pooled genomic libraries were then sequenced using the Illumina iSeq 100 platform and using the single-end sequencing method.

\section{Bioinformatics}

Analysis was done using QIIME 2 2018.11 [21]. Raw FASTA files were quality filtered and denoised using DADA2 [22] through the q2-dada2 plugin. This involved clipping off of illumina associated adaptor and barcode sequences, followed by trimming of low-quality ends [at 200 base pairs (bp)] as well as removal of low-quality bases. All the sequences were then aligned to the MAFFT [23] and the aligned sequences used to construct a rooted phylogenetic tree using fasttree2 [24] via q2-phylogeny. We then rarefied the samples to 28,076 sequences per sample and estimated alpha diversity metrics, beta diversity metrics and Principle Coordinate Analysis ( $\mathrm{PCoA}$ ) using q2-diversity. The alpha diversity metrics included; Observed operational taxonomic units (OTUs), Evenness, Shannon diversity [25] and Faith's Phylogenetic Diversity [26] while the beta diversity distance matrices included unweighted UniFrac [27], weighted UniFrac [28] and Bray-Curtis dissimilarity [29]. The estimated beta diversity between communities was then visualised using principal coordinate analysis ( $\mathrm{PCoA}$ ) plots. To assign taxonomy to the amplicon sequence variants (ASVs), we used the q2-feature-classifier [30] basing on the classify-sklearn naive Bayes taxonomy classifier with the Greengenes 13_8 99\% OTU's as reference sequences [31]. We assessed the progress of development within the microbiota composition from 11 days through weaning to 63 days of age using q2-longitudinal [32]. Using PICRUSt (phylogenetic Investigation of Communities by Reconstruction of Unobserved States) [16] we predicted KEGG orthology (KO) metagenomes, enzyme commission (EC) metagenomes and MetaCyc pathway abundances through the $\mathrm{q} 2$ picrust2 plugin.

\section{Statistical analysis}

The data from QIIME 2 was imported into R statistical software [33] for statistical analysis using vegan [34] and Bioconductor packages. To test for statistically significant associations between ADG and relative abundance of genera or pathway abundances, we fit a generalized linear regression using the edgeR package [35]. We corrected for multiple hypothesis testing using the false discovery rafe (FDR) correction method.

\section{Sequence data accession number}

All sequence data in this study has been deposited in the NCBI Sequence Read Archive (SRA) database under the BioProject accession number PRJNA602130.

\section{RESULTS}

\section{Sequencing data}

The twenty-four samples sequenced yielded a total of 3,212,205 sequences ranging from 4 to $284,317$ sequences per sample (mean $=133,841.875$ and median $=164,482)$. Quality control and subsequent removal of poor-quality reads in QIIME2 reduced the sequences to a total of 2,390,641 sequences with a mean of 99,610 sequences per sample (ranging from 3-243,908 sequences while median $=117,350.5)$. 


\section{Diversity}

In order to create an even subsample from each of the samples for calculation of diversity, a sub sampling depth of 28,076 was used. This eliminated 2 samples (B1P2 and B1P5) with fewer reads and left 22 samples with a total of 617,672 reads that were then used to calculate alpha and beta diversities among the samples. The rarefaction plot based on the observed OTU's (Fig. S1) showed a trajectory that initially steeply increased before levelling off at around 40,000 sequences. This indicated that there was good coverage of the communities among all samples.

\section{Taxonomic analysis}

The sequences were then clustered into 1,848 OTUs. Taxonomy was assigned by aligning the sequences against the Greengenes 16S rRNA data base at 99\% similarity using a Naive Bayes classifier trained on the V4 hypervariable region. The sequences were dominated by members of the kingdom Bacteria except for two genera belonging to the kingdom Archaea. Proteobacteria and Firmicutes were the most dominant phyla, with a combined relative abundance ranging from 53.17\%-97.4\% (Fig. 1A). Streptococcaceae, Pasteurellaceae, Moraxellaceae, Veillonellaceae, Neisseriaceae, Leptotrichiaceae, and Lachnospiraceae were the most represented families (Fig. 1B). Lactobacillaceae tended to increase in relative abundance post weaning. The most prevalent genera within the samples were Streptococcus (Firmicutes) Actinobacillus (Proteobacteria), Moraxella (Proteobacteria), Veillonella (Firmicutes) and Haemophilus (Proteobacteria) (Fig. 1C).

\section{Development of the oropharyngeal microbial community}

In order to study the broad changes in microbiota during development, samples were collected at 11,26 , and 63 days of age. Within the first 11 days of life, the piglets' diet was composed entirely of sow milk and creep feeding was initiated at day 12 of life. The samples collected on the 26th day of life represent the oropharyngeal microbial community at the end of nursing. While the samples collected at 63 days of life represented the oropharyngeal microbiota before the end of the weaner stage of life.

PCoA showed that the samples already clustered closely by day 11 of age regardless of maternal or foster sow, that nursed them. Pena Cortes et al. [36] reported a litter effect in the tonsillar microbiota that disappeared by the third week. We did not observe this litter effect by the 11th day of life, probably due to the advancement in age of the piglets in our study compared to those in their study [36]. Further, the samples tended to clusters by age of the piglets except for 2 piglets at 26 days of age, that had been weaned 6 days earlier (Fig. 2). The divergence of the weaned piglets microbiota was expected since weaning stress has been found to disrupt the tonsillar microbial composition [37].

In order to investigate the development pattern influenced by birthweight, we assessed the longitudinal changes in alpha and beta diversity between the birthweight groups (high birthweight, HBW and low birthweight, LBW groups). We found that the Shannon diversity tended to decline between 11 and 26 days of age in both groups of piglets and gradually increased between 26 and 63 days of age (Fig. 3A). Furthermore, we found that the beta diversity tended to change faster in HBW than the LBW piglets during suckling (between 11 and 26 days) (Fig. 3B), however by 63 days of age, the microbiota composition in both groups was relatively equally different from their relative compositions at 11 days of age (Fig. 3C).

\section{Associations between the microbiota and ADG}

In order to test whether there was an association between the oropharyngeal microbiota and the ADG of piglets, we run a generalized linear regression using the edgeR package in R. We found 9 genera that were significantly associated with $\mathrm{ADG}$ at 11 days of age $(\mathrm{FDR}<0.05)$ and 9 genera 
A

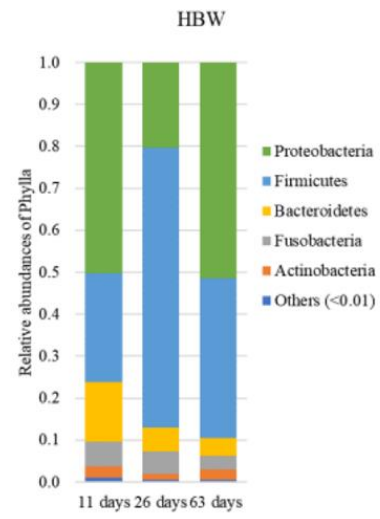

B

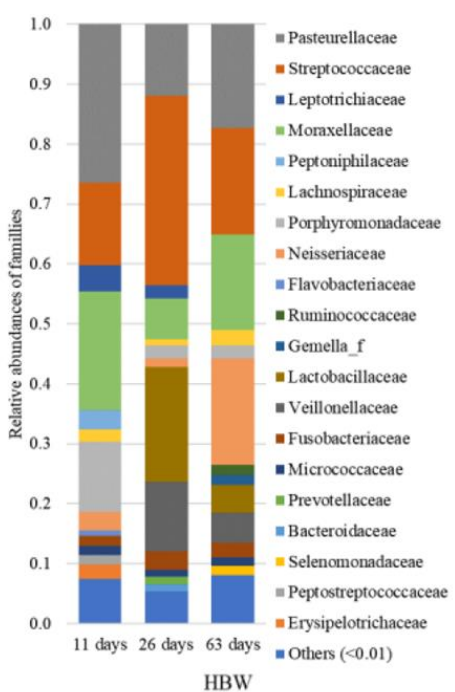

C

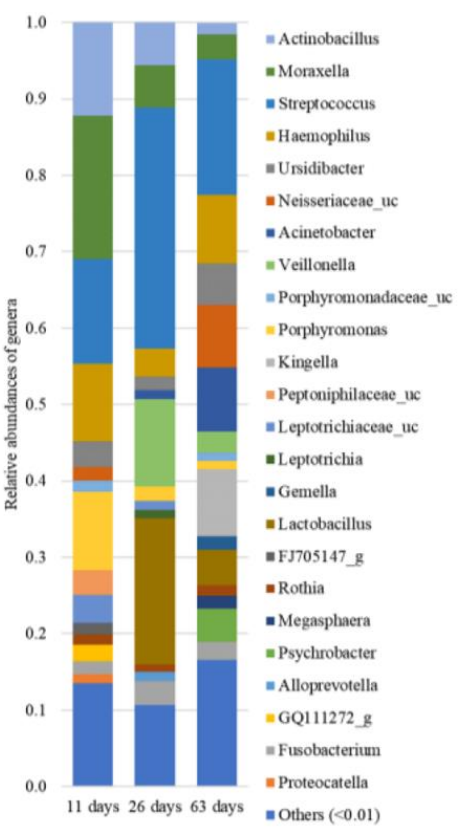

LBW
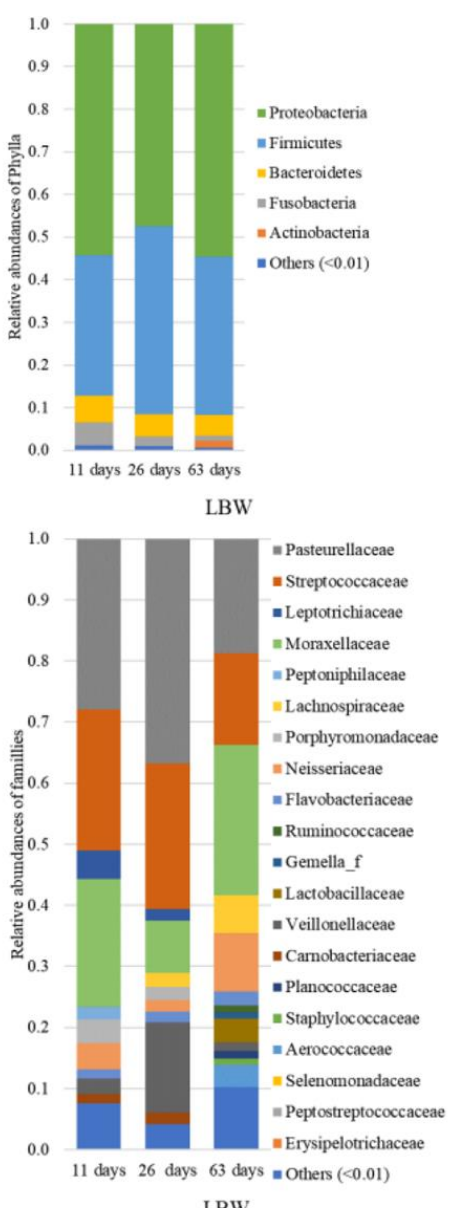

LBW

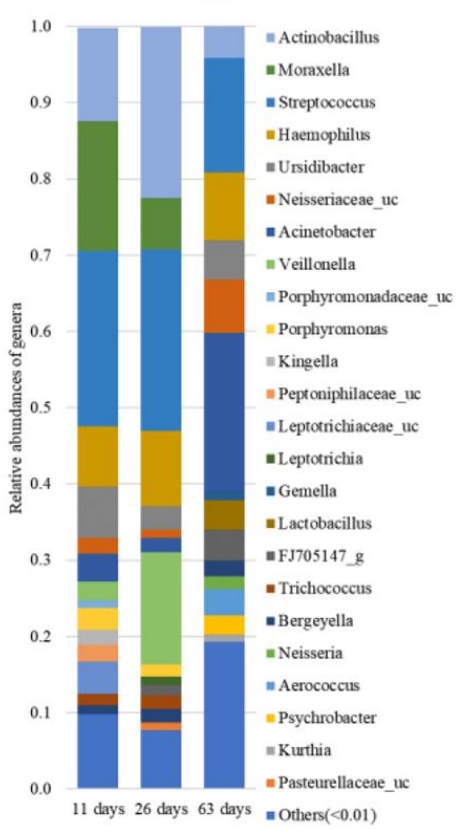

Fig. 1. Stacked bar graphs showing the phylogenetic composition of the oropharyngeal microbiota in high birthweight (HBW) vs low birthweight piglets (LBW) at 11, 26, and 63 days of age. The comparison is made in $(A)$ at phylum level, $(B)$ at family level and $(C)$ at genus level. 


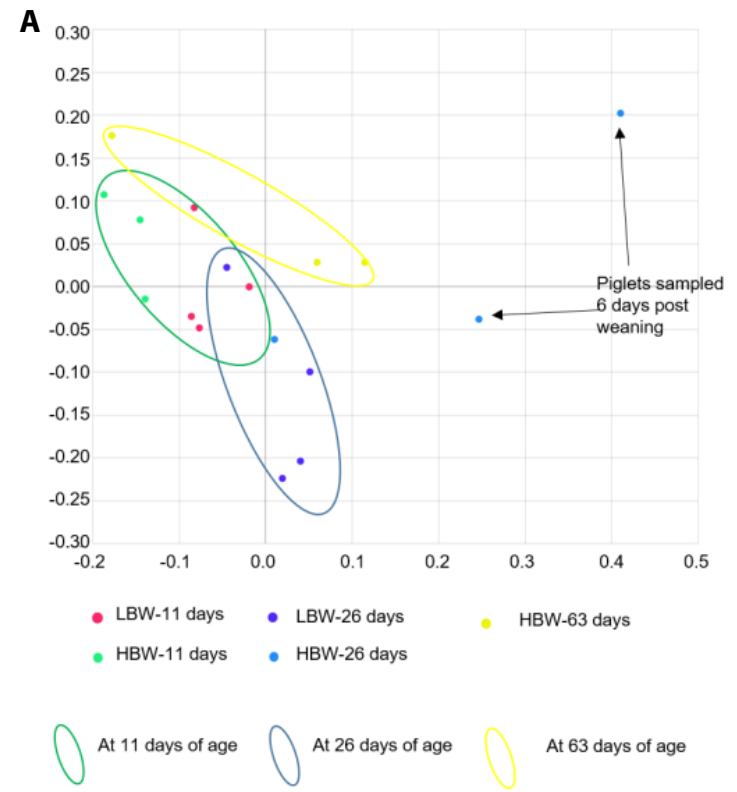

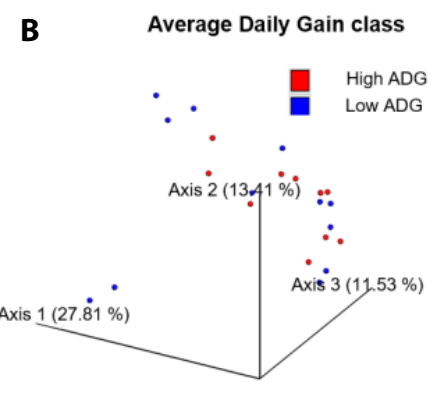

Age at sampling

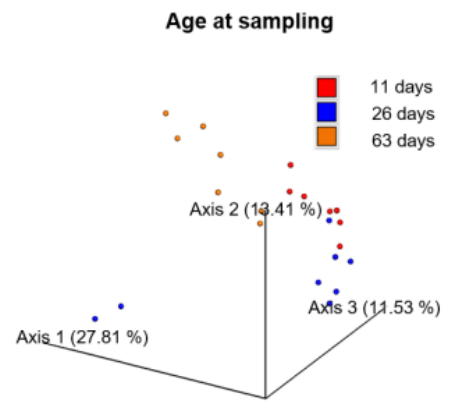

Body weight class

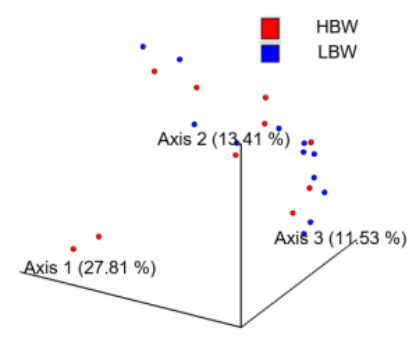

Age at weaning

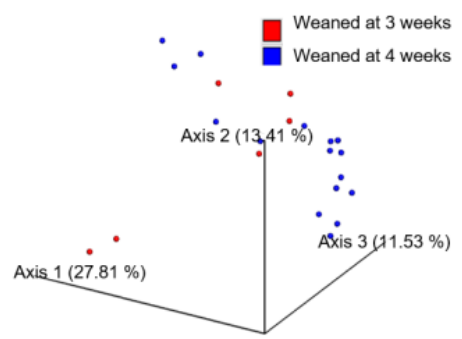

Fig. 2. Principle Coordinate Analysis (PCoA) plots illustrating the phylogenetic composition of the piglets' oropharyngeal microbial community. (A) Two dimensional PCoA plot based on unweighted UniFrac distances showing the distribution of the microbiota and $95 \%$ distribution ellipses at the 3 time points. (B) Three dimensional PCoA plots based on Bray Curtis distances showing the distribution of the oropharyngeal microbiota in samples collected at 11 , 26 , and 63 days of age. The symbols represent data from individual piglets and are color-coded by the indicated categories of metadata.

A

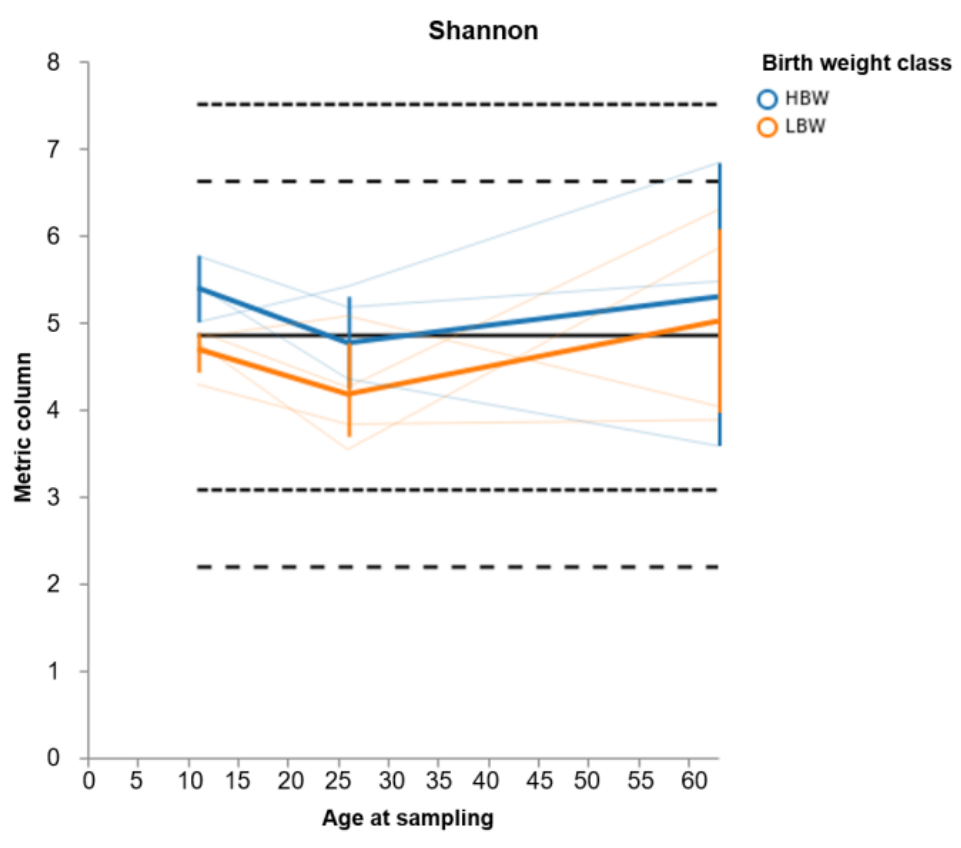

B

Pairwise Distances between 11 and 26 days

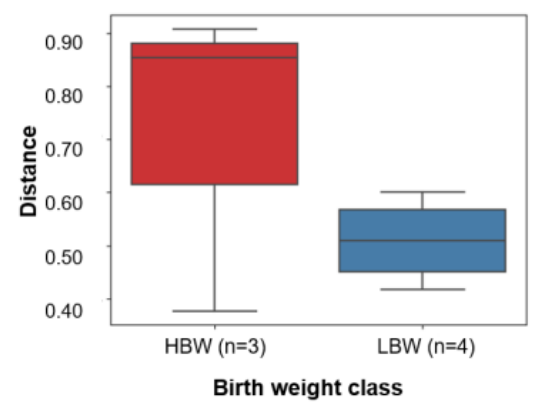

C

Pairwise Distances between 11 and 63 days

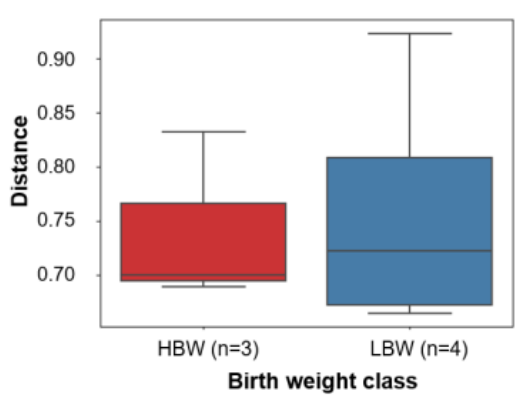

Fig. 3. Longitudinal analysis of microbial composition. In $(A)$ is a volatility plot of Shannon diversity showing changes in alpha diversity in high birth weight (HBW) and in low birth weight (LBW) piglets over the study period. In (B-C) are boxplots showing the paired distances in beta diversity of HBW and LBW piglets; (B) between 11 and 26 days of age and $(C)$ between 11 and 63 days of age. The distances are based on the Bray Curtis distance matrices between each subject's diversity at the given time points. 
(FDR < 0.1 ) at 26 days of age (Table 1 and 2). However, at 63 days of age, no genera were found to be associated with ADG. The association between the microbiota and ADG was stronger earlier in life and reduced post weaning implying that it is potentially more effective to influence weight gain

Table 1. Genera that were significantly associated with ADG at 11 days of age

\begin{tabular}{|c|c|c|c|c|c|c|}
\hline & Phylum;Class;Order;Family;Genus & $\log \mathrm{FC}$ & $\log C P M$ & LR & $p$-value & FDR \\
\hline 1 & Firm;Nega;Vell;Veil;Veillonella & -14.67782 & 16.60199 & 15.9995 & $6.34 \mathrm{E}-05$ & 0.004270 \\
\hline 2 & Firm;Baci;Lact;Aero;Aerococcus & 26.99785 & 14.38829 & 15.87192 & $6.78 \mathrm{E}-05$ & 0.004270 \\
\hline 3 & Bact;Flav;Flav;[Wee];Bergeyella & -37.34028 & 12.99741 & 14.98349 & 0.000108 & 0.004377 \\
\hline 4 & Bact;Bact;Bact;[Par];[Prevotella] & -18.82124 & 13.47373 & 14.85977 & 0.000116 & 0.004377 \\
\hline 5 & Prot;Gamm;Pseu;Mora;Psychrobacter & 49.12427 & 13.21755 & 13.95362 & 0.000187 & 0.005902 \\
\hline 6 & Firm;Baci;Baci;Plan;Kurthia & 24.61041 & 8.322315 & 10.81635 & 0.001006 & 0.027109 \\
\hline 7 & Firm;Baci;Lact;Ente;Enterococcus & 35.18011 & 9.889504 & 10.57308 & 0.001147 & 0.027109 \\
\hline 8 & Prot;Gamm;Pseu;Mora;Acinetobacter & 18.29274 & 17.07745 & 10.1381 & 0.001452 & 0.030500 \\
\hline 9 & Firm;Baci;Lact;Aero;Facklamia & 19.61991 & 10.15351 & 9.796667 & 0.001748 & 0.033043 \\
\hline 10 & Prot;Gamm;Past;Past;Haemophilus & -9.712501 & 14.02468 & 8.132637 & 0.004348 & 0.068474 \\
\hline 11 & Firm;Baci;Baci;Plan; & 39.45043 & 10.27581 & 7.677588 & 0.005591 & 0.074895 \\
\hline 12 & Bact;Bact;Bact;[Par]; & -27.74231 & 9.776943 & 7.637212 & 0.005718 & 0.074895 \\
\hline 13 & Prot;Gamm;Past;Past;Pasteurella & -10.47806 & 11.74235 & 7.567197 & 0.005944 & 0.074895 \\
\hline
\end{tabular}

Nine genera (Veillonella, Aerococcus, Bergeyella, Prevotella, Psychrobacter, Kurthia, Enterococcus, Acinetobacter and Facklamia) within the oropharyngeal microbiota were significantly associated with $A D G$ at 11 days of age $(F D R<0.05)$.

[]; The names in square brackets [ ] within the Greengenes database are still contested at that taxonomic level.

ADG, average daily gain; logFC, log fold-change; logCPM, log counts per million; LR, likelihood ratio; FDR, false discovery rate (adjusted $p$-value).

Phylla; Cyan - Cyanobacteria, Firm - Firmicutes, Bact - Bacteroidetes, Prot - Proteobacteria. Classes; Nega - Negativicutes, Baci - Bacilli, Flav - Flavobacteriia, Bact - Bacteroidia, Gamm - Gammaproteobacteria, Alph- Alphaproteobacteria. Orders; Vell - Vellionellales, Lact - Lactobacilliales, Flav - Flavobacteriales, Bact - Bacteroidales, Pseu - Pseudomonadales, Baci - Bacillales, Lact - Lactobacillales, Past - Pasteurellales. Families; Veil - Veillonellaceae, Aero - Aerococcaceae, Mora - Moraxellaceae, Past - Pasteurellaceae, Plan - Planococcaceae, Past - Pasteurellaceae, Ente - Enterococcaceae.

Table 2. Genera that were significantly associated with ADG at 26 days of age

\begin{tabular}{rlrrrcc}
\hline & \multicolumn{1}{c}{ Phylum;Class;Order;Family;Genus } & logFC & logCPM & LR & p-value & FDR \\
\hline 1 & Firm;Clos;Clos;[Tis];Helcococcus & -23.44355 & 14.17177 & 12.71057 & 0.000364 & 0.053548 \\
2 & Acti;Acti;Acti;Derm;Helcobacillus & -25.88382 & 7.692197 & 11.72411 & 0.000617 & 0.053548 \\
3 & Firm;Baci;Lact;Aero;Aerococcus & 19.01275 & 14.38829 & 11.12881 & 0.00085 & 0.053548 \\
4 & Bact;Bact;Bact;; & -14.66345 & 15.57125 & 9.673499 & 0.001869 & 0.088331 \\
5 & Fuso;Fuso;Fuso;Lept; & -13.97292 & 14.54914 & 9.123524 & 0.002523 & 0.094253 \\
6 & Firm;Erys;Erys;Erys;;1 & -16.66539 & 12.16592 & 8.465524 & 0.003619 & 0.094253 \\
7 & Firm;Baci;Lact;Leuc;Weissella & 16.41927 & 9.654118 & 8.248555 & 0.004078 \\
8 & Acti;Acti;Act;:Diet;Dietzia & 33.5106 & 7.878502 & 8.101699 & 0.004422 & 0.094253 \\
9 & Acti;Acti;Acti;Acti;Arcanobacterium & -29.88175 & 10.98488 & 8.07491 & 0.004488 & 0.094253 \\
10 & Prot;Gamm;Pseu;Mora;Psychrobacter & 19.90006 & 13.21759 & 7.650028 & 0.005677 & 0.107298 \\
\hline
\end{tabular}

Nine genera (Helcococcus, Helcobacillus, Aerococcus, Weissella, Dietzia, Arcanobacterium, and 3 unidentified genera) within the oropharyngeal microbiota were significantly associated with ADG at 26 days of age (FDR < 0.1$)$. Because 3 sequence data of $16 S$ RNA have not been identified in the phylogenetic level of genus in the Greengenes database, they are remarked as 3 unidentified genera.

ADG, average daily gain; logFC, log fold-change; logCPM, log counts per million; LR, likelihood ratio; FDR, false discovery rate (adjusted $p$-value).

[]; The name in square bracket [ ] within the Greengenes database is still contested at that taxonomic level.

Phylla; Firm - Firmicutes, Acti - Actinobacteria, Bact - Bacteroidetes, Fuso - Fusobacteria, Prot - Proteobacteria Classes; Clos- Clostridia, Acti - Actinobacteria, Baci - Bacilli, Bact - Bacteroidia, Fuso - Fusobacteriia, Erys - Erysipelotrichia, Gamm - Gammaproteobacteria. Orders; Clos- Clostridiales, Acti - Actinomycetales, Lact - Lactobacilliales, Bact - Bacteroidales, Fuso - Fusobacteriales, Erys - Erysipelotrichiales, Pseu - Pseudomonadales. Families; Derm - Dermabacteraceae, Aero - Aerococcaceae, Lept - Leptotrichiaceae, Erys Erysipelotrichaceae, Leuc - Leuconostocaceae, Diet - Dietziaceae, Acti - Actinomycetaceae, Mora - Moraxellaceae. 
by manipulating the microbiota earlier in pre-weaning life of the piglets.

\section{Associations between the predicted microbial functional profile and ADG}

The association with ADG might be explained better by the metabolic functions performed by the microbial communities in the piglets with diverging ADG's. To investigate this, we used q2picrust2, which uses $16 \mathrm{~S}$ rRNA gene sequence data to predict the microbial functional repertoire within the samples based on curated reference databases such as the KEGG [38] and MetaCyc [39]. The output from q2picrust2 analysis included predictions of 6,490 $\mathrm{KO}$ metagenomes based on the KEGG's Orthologs, 1,980 EC metagenomes and 389 MetaCyc pathway abundances within the 24 samples.

PCoA was used to show the distribution of samples based on the Bray-Curtis distances within their composition of $\mathrm{KO}$ metagenomes, $\mathrm{EC}$ metagenomes and MetaCyc pathway abundances (Fig. S2). From the PCoA plots it could be seen that weaning had the strongest effect on microbial functional profile and that the samples tended to cluster by age.

We used the MetaCyc pathways output from q2picrust2 to identify metabolic pathways that were associated with ADG in the piglets. To do this we again used a generalized linear regression model within the edgeR package in R. We found 45, 10, and 2 metabolic pathways that were significantly associated with ADG at 11,26, and 63 days of age, respectively (FDR < 0.05 ). The MetaCyc pathways found to be significantly associated with ADG were cross referenced with the MetaCyc catalogue [39] to identify their names and the super pathway categories to which they belonged (Tables 3-5).

The pathways associated with ADG belonged to the following categories of super pathways; aromatic compound degradation; nucleoside and nucleotide biosynthesis; nucleoside and nucleotide degradation; sugar nucleotide biosynthesis; sugar acid metabolism, Vitamin $\mathrm{K}_{2}$ biosynthesis; amino acid biosynthesis; amino acid degradation; amine and polyamine degradation; amine and polyamine biosynthesis; Secondary metabolite degradation; Cell structure biosynthesis; polymyxin resistance; Fatty acid and lipid biosynthesis; Fatty acid Lipid degradation; Inorganic Nutrient Metabolism; Cofactor, prosthetic group, electron carrier and Vitamin biosynthesis; Generation of precursor metabolite and energy; aldehyde degradation; sugar biosynthesis and Antibiotic resistance (Polymyxin resistance) (Tables 3-5).

The aromatic compound degradation category included pathways that are involved in the degradation of Catechol. Apart from the breakdown of xenobiotics, these pathways are capable of degrading the catechol residue derived from the host's catecholamines hormones. Within the nucleoside and nucleotide metabolism pathways, we found that pathways involved in biodegradation significantly increased with ADG during the pre-weaning stage (11 and 26 days of age) while their biosynthesis significantly reduced with increasing ADG (Table 3 and 4). In the Amine and polyamine degradation category, pathways involved in the degradation of allantoin and aromatic biogenic amines increased with ADG while biosynthesis of ectoine decreased with ADG (Table 3). Allantoin is a by-product of purine degradation and therefore it's degradation is connected to the microbes' degradation of nucleotides and nucleosides as a nitrogen source [40,41]. We also observed an increased representation of pathways involved in the degradation of D-glucarate and D-galactarate in high ADG piglets implying that organisms associated with high rates of weight gain have the ability to degrade sugar acids and utilize them as a carbon source for growth (Table 3 and 4).

\section{DISCUSSION}

This study investigated the association between the oropharyngeal microbiota and the piglets' birth- 
Table 3. Metabolic pathways that were significantly associated with ADG at 11 days of age

\begin{tabular}{|c|c|c|c|c|c|c|c|}
\hline & Super pathway & Pathway & $\log F C$ & $\log C P M$ & LR & $p$-value & FDR \\
\hline 1 & $\begin{array}{l}\text { Nucleoside and nucleotide } \\
\text { biosynthesis }\end{array}$ & Super pathway of pyrimidine nucleobases salvage & -0.839 & 13.071 & 29.653 & $5.17 \mathrm{E}-08$ & 2.01E-05 \\
\hline 2 & $\begin{array}{l}\text { Generation of precursor metabolite } \\
\text { and Energy }\end{array}$ & $\begin{array}{l}\text { Pentose phosphate pathway } \\
\text { (non-oxidative branch) }\end{array}$ & -0.824 & 13.094 & 20.929 & 4.77E-06 & 0.000590 \\
\hline 3 & Aromatic compound degradation & $\begin{array}{l}\text { Toluene degradation II (aerobic) } \\
\text { (via 4-methylcatechol) }\end{array}$ & 16.045 & 8.814 & 20.366 & $6.40 \mathrm{E}-06$ & 0.000590 \\
\hline 4 & Aromatic compound degradation & Toluene degradation I (aerobic) (via o-cresol) & 16.044 & 8.814 & 20.323 & $6.54 \mathrm{E}-06$ & 0.000590 \\
\hline 6 & Amine and polyamine degradation & Allantoin degradation to glyoxylate III & 14.748 & 7.650 & 16.166 & $5.80 \mathrm{E}-05$ & 0.003504 \\
\hline 7 & Inorganic nutrient metabolism & Nitrate reduction VI (assimilatory) & -7.340 & 9.503 & 16.009 & $6.30 \mathrm{E}-05$ & 0.003504 \\
\hline 8 & Sugar acid metabolism & D-Galactarate degradation I & 8.898 & 4.542 & 14.948 & 0.000111 & 0.005055 \\
\hline 9 & Sugar acid metabolism & D-Glucarate and D-galactarate degradation & 8.898 & 4.542 & 14.841 & 0.000117 & 0.005055 \\
\hline 12 & Aromatic compound degradation & Catechol degradation to $\beta$-ketoadipate & 28.386 & 8.809 & 14.070 & 0.000176 & 0.005294 \\
\hline 13 & Fatty acid and lipid biosynthesis & Gondoate biosynthesis (anaerobic) & -0.793 & 13.048 & 13.932 & 0.000190 & 0.005294 \\
\hline 14 & $\begin{array}{l}\text { Nucleoside and } \\
\text { nucleotide degradation }\end{array}$ & Adenosine nucleotides degradation II & 9.844 & 9.195 & 13.793 & 0.000204 & 0.005294 \\
\hline 15 & Amino acid degradation & L-Tyrosine degradation I & 12.429 & 8.600 & 13.747 & 0.000209 & 0.005294 \\
\hline 16 & Amine and polyamine degradation & Aromatic biogenic amine degradation (bacteria) & 19.374 & 6.380 & 13.671 & 0.000218 & 0.005294 \\
\hline 17 & Aromatic compound degradation & Super pathway of salicylate degradation & 26.786 & 8.625 & 13.487 & 0.000240 & 0.005497 \\
\hline 18 & Amino acid degradation & L-Leucine degradation I & 12.250 & 9.407 & 13.213 & 0.000278 & 0.005952 \\
\hline 19 & $\begin{array}{l}\text { Nucleoside and } \\
\text { nucleotide degradation }\end{array}$ & Purine nucleotides degradation II (aerobic) & 8.390 & 9.712 & 13.129 & 0.000291 & 0.005952 \\
\hline 25 & Aromatic compound degradation & Meta cleavage pathway of aromatic compounds & 22.121 & 6.813 & 11.049 & 0.000888 & 0.013810 \\
\hline 26 & Aromatic compound degradation & $\begin{array}{l}\text { 3-Phenylpropanoate and 3-(3-hydroxyphenyl) } \\
\text { propanoate degradation }\end{array}$ & 27.451 & 8.520 & 10.975 & 0.000924 & 0.013817 \\
\hline 27 & Secondary metabolite degradation & $\begin{array}{l}\text { Engineered pathway: isoprene biosynthesis II } \\
\text { (engineered) }\end{array}$ & 12.333 & 4.357 & 10.740 & 0.001048 & 0.015103 \\
\hline 28 & $\begin{array}{l}\text { Nucleoside and } \\
\text { nucleotide biosynthesis }\end{array}$ & Adenosine ribonucleotides de novo biosynthesis & -0.603 & 12.960 & 10.266 & 0.001355 & 0.018827 \\
\hline 29 & $\begin{array}{l}\text { Nucleoside and } \\
\text { nucleotide biosynthesis }\end{array}$ & $\begin{array}{l}\text { Super pathway of adenosine nucleotides } \\
\text { de novo biosynthesis I }\end{array}$ & -0.693 & 12.986 & 10.005 & 0.001561 & 0.020945 \\
\hline 30 & Aromatic compound degradation & 4-Methylcatechol degradation (ortho cleavage) & 27.042 & 8.601 & 9.596 & 0.001950 & 0.024940 \\
\hline 31 & Aromatic compound degradation & Catechol degradation II (meta-cleavage pathway) & 12.723 & 6.818 & 9.561 & 0.001988 & 0.024940 \\
\hline 32 & Aromatic compound degradation & $\begin{array}{l}\text { Protocatechuate degradation II (ortho-cleavage } \\
\text { pathway) }\end{array}$ & 10.463 & 8.309 & 9.373 & 0.002202 & 0.026766 \\
\hline 33 & Sugar nucleotide biosynthesis & CMP-legionaminate biosynthesis I & -8.205 & 8.984 & 9.189 & 0.002434 & 0.028639 \\
\hline 34 & Aromatic compound degradation & Phenylacetate degradation I (aerobic) & 20.830 & 8.727 & 9.138 & 0.002503 & 0.028639 \\
\hline 35 & Aldehyde degradation & Super pathway of methylglyoxal degradation & 21.047 & 6.569 & 8.790 & 0.003029 & 0.033661 \\
\hline 36 & Sugar biosynthesis & Sucrose biosynthesis III & 18.218 & 4.108 & 8.710 & 0.003165 & 0.034199 \\
\hline 37 & Amine and polyamine biosynthesis & Ectoine biosynthesis & 15.114 & 3.587 & 8.505 & 0.003543 & 0.037244 \\
\hline 38 & Amino acid degradation & L-arginine degradation II (AST pathway) & 12.522 & 4.822 & 8.097 & 0.004434 & 0.045390 \\
\hline
\end{tabular}


Table 3. Continued

\begin{tabular}{lllrrrrr}
\hline \multicolumn{1}{c}{ Super pathway } & \multicolumn{1}{c}{ Pathway } & logFC & logCPM & LR & \multicolumn{1}{c}{$\boldsymbol{p}$-value } & FDR \\
\hline 39 & Cell structure biosynthesis & Peptidoglycan biosynthesis II (staphylococci) & 4.900 & 9.105 & 7.908 & 0.004922 & 0.048418 \\
40 & $\begin{array}{l}\text { Nucleoside and nucleotide } \\
\text { biosynthesis }\end{array}$ & Super pathway of adenosine nucleotides de novo & -0.697 & 12.922 & 7.853 & 0.005073 & 0.048418 \\
41 & Cell structure biosynthesis & $\begin{array}{l}\text { UDP-N-acetylmuramoyl-pentapeptide biosynthesis I } \\
\text { (meso-diaminopimelate containing) }\end{array}$ & -0.739 & 12.817 & 7.781 & 0.005280 & 0.048418 \\
42 & Secondary metabolite degradation & Sulfoquinovose degradation I & 10.149 & 3.185 & 7.742 & 0.005395 & 0.048418 \\
43 & Aromatic compound degradation & Catechol degradation to 2-hydroxypentadienoate II & 11.213 & 6.438 & 7.732 & 0.005425 & 0.048418 \\
44 & Amino acid biosynthesis & L-Lysine biosynthesis II & -2.476 & 11.855 & 7.715 & 0.005477 & 0.048418 \\
45 & Antibiotic resistance & Polymyxin resistance & 9.731 & 3.860 & 7.668 & 0.005621 & 0.048588 \\
\hline
\end{tabular}

ADG, average daily gain; logFC, log fold-change; logCPM, log counts per million; LR, likelihood ratio; FDR, false discovery rate (adjusted $p$-value).

Table 4. Metabolic pathways that were significantly associated with ADG at 26 days of age

\begin{tabular}{|c|c|c|c|c|c|c|c|}
\hline & Super pathway & Pathway & $\log F C$ & $\log C P M$ & LR & $p$-value & FDR \\
\hline 1 & Aromatic compound degradation & $\begin{array}{l}\text { Catechol degradation I } \\
\text { (meta-cleavage pathway) }\end{array}$ & 15.28376 & 8.613798 & 19.28901 & 1.12E-05 & 0.00437 \\
\hline 2 & Aromatic compound degradation & Catechol degradation to 2-hydroxypentadienoate II & 12.99083 & 6.437917 & 15.3016 & 9.16E-05 & 0.0148 \\
\hline 3 & Aromatic compound degradation & $\begin{array}{l}\text { Catechol degradation II } \\
\text { (meta-cleavage pathway) }\end{array}$ & 12.94294 & 6.817753 & 14.8872 & 0.000114 & 0.0148 \\
\hline 4 & Fatty acid and lipid biosynthesis & Super pathway of lipopolysaccharide biosynthesis & 28.38013 & 2.42697 & 13.50893 & 0.000237 & 0.02309 \\
\hline 5 & Aromatic compound degradation & $\begin{array}{l}\text { Toluene degradation II (aerobic) } \\
\text { (via 4-methylcatechol) }\end{array}$ & 10.58863 & 8.813538 & 12.74445 & 0.000357 & 0.023322 \\
\hline 6 & Aromatic compound degradation & $\begin{array}{l}\text { Toluene degradation I (aerobic) } \\
\text { (via o-cresol) }\end{array}$ & 10.58859 & 8.813538 & 12.73057 & 0.00036 & 0.023322 \\
\hline 7 & Sugar acid metabolism & D-Galactarate degradation I & 8.848847 & 4.542388 & 11.51003 & 0.000692 & 0.031156 \\
\hline 8 & Antibiotic resistance & Polymyxin resistance & 15.29624 & 3.859829 & 11.46961 & 0.000707 & 0.031156 \\
\hline 9 & Sugar acid metabolism & $\begin{array}{l}\text { Super pathway of D-glucarate and } \\
\text { D-Galactarate degradation }\end{array}$ & 8.848833 & 4.542388 & 11.43476 & 0.000721 & 0.031156 \\
\hline 10 & Nucleoside and nucleotide biosynthesis & Super pathway of pyrimidine nucleobases salvage & -0.46889 & 13.07049 & 10.52772 & 0.001176 & 0.045745 \\
\hline 11 & Sugar acid metabolism & D-glucarate degradation I & 8.936948 & 4.805885 & 10.33191 & 0.001307 & 0.046238 \\
\hline 12 & Amine and polyamine degradation & Allantoin degradation IV (anaerobic) & 12.94646 & 6.715548 & 9.796436 & 0.001749 & 0.051378 \\
\hline
\end{tabular}

$A D G$, average daily gain; logFC, log fold-change; logCPM, log counts per million; LR, likelihood ratio; FDR, false discovery rate (adjusted $p$-value).

Table 5. Metabolic pathways that were significantly associated with ADG at 63 days of age

\begin{tabular}{|c|c|c|c|c|c|c|c|}
\hline & Super pathway & Pathway & $\log \mathrm{FC}$ & $\log$ CPM & LR & $p$-value & FDR \\
\hline 1 & Vitamin $\mathrm{K}_{2}$ biosynthesis & 1,4-Dihydroxy-6-naphthoate biosynthesis I & 43.60243 & 5.323691 & 13.81177 & 0.000202 & 0.041719 \\
\hline 2 & Inorganic nutrient metabolism & $\begin{array}{l}\text { Super pathway of sulphur oxidation } \\
\text { (Acidianus ambivalens) }\end{array}$ & 27.23389 & 8.525369 & 13.69964 & 0.000214 & 0.041719 \\
\hline 3 & Fatty acid and lipid degradation & Phospholipase pathway & -48.3973 & -0.97698 & 12.20301 & 0.000477 & 0.061867 \\
\hline 4 & Vitamin $\mathrm{K}_{2}$ biosynthesis & 1,4-Dihydroxy-6-naphthoate biosynthesis II & 28.17554 & 6.774948 & 10.42818 & 0.001241 & 0.120694 \\
\hline 5 & Aromatic compound degradation & Catechol degradation to 2-hydroxypentadienoate II & 17.00923 & 6.437778 & 7.659094 & 0.005649 & 0.407778 \\
\hline
\end{tabular}

ADG, average daily gain; logFC, log fold-change; logCPM, log counts per million; LR, Likelihood ratio; FDR, False discovery rate (adjusted $p$-value).

weight and rate of weight gain. Results revealed microbial clades and pathways that were associated with the piglets' weight gain. However, the associations were stronger in the pre-weaning phase and appeared to wane with age implying that an attempt to improve weight gain through modulation of the microbiota should target early life of the piglet, particularly before 4 weeks of life.

Our findings from the taxonomic analysis of the oropharyngeal microbiota were largely in 
agreement with studies focusing on the tonsillar microbiota [36,42,43]. However, the dominance of Streptococcus, in our study, was striking since it was only represented as a minor community member within the tonsils [42]. It has been found that the catecholamine stress hormones, norepinephrine (NOR) and dopamine (DOP), induce the proliferation of some species of Streptococcus [44]. In our study, the piglets were raised under a commercial farm setting, which is characterized by cross fostering, weaning and frequent regrouping of the piglets. These events involve changing the piglets environment and disruption of their social structure, consequently elevating the levels of circulating catecholamines [45]. In addition, Pasteurella, which was found to dominate in the tonsillar microbial communities [42] had a surprisingly low relative abundance among our samples ( $1 \%$ or less). These slight differences can partly be explained by the fact that the above studies focused on the tonsillar microbiota while this study included pooled samples of the oropharyngeal cavity.

We did not find any statistically significant difference between the development of oropharyngeal microbiota in HBW and LBW piglets. However, we found subtle differences that tended to disappear by 63 days of life implying that birthweight only had a slight effect on the oropharyngeal microbiota during the first few weeks of pre-weaning which gradually wanes as the piglets grow older. In contrast to our findings, studies done in the piglets' gut have found statistically significant differences between the microbiota of low birthweight and normal birthweight piglets during the pre-weaning phase $[46,47]$. However, these differences also tended to disappear by 35 days of age [46].

The current trend in microbiome research has been to explore the microbial metabolic functions that may have clinical, therapeutic or nutritional relevance to the host [48]. Previous studies in domestic pigs have reported correlations between feed efficiency and the illeal, caecal, colonic and fecal microbiome [49-51]. In this study, we explored the association between the predicted metabolic pathways within the oropharyngeal microbiome and ADG in the piglets. Our results showed an enrichment of pathways involved in the biodegradation of nucleosides and nucleotides in heavier piglets during the pre-weaning phase. The reason for this association is not clear, however one possible explanation could be, that the relationship is a consequence, and not a cause, of the ADG among the piglets. It is known that sow milk, like all mammalian milk is a very rich source of nucleosides [52]. It is therefore possible that, as a result of their competitive advantage, the larger piglets have more access to the best teats and also spend more time suckling [53] resulting in a consistently higher concentration of sow milk-derived nucleosides within the saliva matrix in their oropharynx. This would then favor the proliferation of bacteria with the ability to utilize nucleosides and nucleotides. On the other hand, members of the oropharyngeal microbiota within the lighter piglets have to biosynthesize their nucleotides and nucleosides to sustain their growth and proliferation.

The association of catechol degradation pathways with ADG, suggests an enhanced ability to degrade host derived catecholamines within the oropharyngeal microbiome of piglets having high rates of weight gain. Catecholamines, which include Dopamine (DOP), Epinephrine (EPI) and Norepinephrine (NOR), are secreted during stressful conditions [54]. It is likely that the high ADG piglets have higher appetite levels compared to the low ADG piglets and are therefore more inclined towards aggression and competition for feed with their pen mates leading to higher levels of stress hormones (EPI and NOR) within their circulation [55,56]. Circulating catecholamines are partly secreted into the saliva matrix and this may lead to an accumulation of bacteria, within the oropharynx, that have the ability to degrade catechols. Catecholamines are also involved in the regulation of hedonic feeding in mammals [57]. Hedonic feeding refers to the non-homeostatic feeding that is not driven by energy requirements but simply driven by pleasure or desire to consume palatable feed [58]. Some catecholamines have been shown to induce feeding behavior such as food-anticipatory activity, search for food and motivation to feed [59]. Breakdown of these 
catecholamines, interferes with systemic regulation of their production [60]. Interfering with the feedback control of the central catecholamine release could then lead to a sustained secretion and consequently, a sustained appetitive feeding in pigs thus contributing to attainment of higher rates of weight gain.

In summary, we identified microbial biomarkers within the oropharyngeal microbiome that may be used as targets for modulating rates of weight gain in piglets. However, our study does not confirm whether the detected microbial signature is a cause or an effect of the ADG therefore, requiring further studies. In future, studies that employ 'omics' techniques are needed in order to better understand the roles played by this oropharyngeal microbiota. Combining techniques that can reveal the active genes (transcriptomics), synthesized protein molecules (proteomics) and their metabolic products (metabolomics) could provide deeper insights into the role of this oropharyngeal microbiome.

\section{SUPPLEMENTARY MATERIALS}

Supplementary materials are only available online from: https://doi.org/10.5187/jast.2020.62.2.247

\section{REFERENCES}

1. Quiniou N, Dagorn J, Gaudré D. Variation of piglets' birth weight and consequences on subsequent performance. Livest Prod Sci. 2002;78:63-70.

2. Douglas SL, Wellock I, Edwards SA, Kyriazakis I. High specification starter diets improve the performance of low birth weight pigs to 10 weeks of age. J Anim Sci. 2014;92:4741-50.

3. Li J. Current status and prospects for in-feed antibiotics in the different stages of pork production: a review. Asian-Australas J Anim Sci. 2017;30:1667-73.

4. Schokker D, Zhang J, Zhang LL, Vastenhouw SA, Heilig HG, Smidt H, et al. Early-life environmental variation affects intestinal microbiota and immune development in new-born piglets. PLoS One. 2014;9:e100040.

5. Sommer F, Bäckhed F. The gut microbiota-masters of host development and physiology. Nat Rev Microbiol. 2013;11:227-38.

6. Turnbaugh PJ, Ley RE, Mahowald MA, Magrini V, Mardis ER, Gordon JI. An obesity-associated gut microbiome with increased capacity for energy harvest. Nature. 2006;444:1027-31.

7. Mikov M. The metabolism of drugs by the gut flora. Eur J Drug Metab Pharmacokinet. 1994;19:201-7.

8. Kim JS, de La Serre CB. Diet, gut microbiota composition and feeding behavior. Physiol Behav. 2018;192:177-81.

9. Mackie RI, Sghir A, Gaskins HR. Developmental microbial ecology of the neonatal gastrointestinal tract. Am J Clin Nutr. 1999;69:1035S-45S.

10. Heinritz SN, Weiss E, Eklund M, Aumiller T, Heyer CM, Messner S, et al. Impact of a highfat or high-fiber diet on intestinal microbiota and metabolic markers in a pig model. Nutrients. 2016;8:317.

11. Thompson CL, Wang B, Holmes AJ. The immediate environment during postnatal development has long-term impact on gut community structure in pigs. ISME J. 2008;2:739-48.

12. Guevarra RB, Hong SH, Cho JH, Kim BR, Shin J, Lee JH, et al. The dynamics of the piglet gut microbiome during the weaning transition in association with health and nutrition.J Anim Sci Biotechnol. 2018;9:54.

13. Yu M, Mu C, Zhang C, Yang Y, Su Y, Zhu W. Marked response in microbial community and 
metabolism in the ileum and cecum of suckling piglets after early antibiotics exposure. Front Microbiol. 2018;9:1166.

14. Shim SB, Verstegen MW, Kim IH, Kwon OS, Verdonk JM. Effects of feeding antibiotic-free creep feed supplemented with oligofructose, probiotics or synbiotics to suckling piglets increases the preweaning weight gain and composition of intestinal microbiota. Arch Anim Nutr. 2005;59:419-27.

15. Liu L, Li Y, Li S, Hu N, He Y, Pong R, et al. Comparison of next-generation sequencing systems.J Biomed Biotechnol. 2012. doi:10.1155/2012/251364.

16. Langille MGI, Zaneveld J, Caporaso JG, McDonald D, Knights D, Reyes JA, et al. Predictive functional profiling of microbial communities using $16 \mathrm{~S}$ rRNA marker gene sequences. Nat Biotechnol. 2013;31:814-21.

17. Mach N, Berri M, Estellé J, Levenez F, Lemonnier G, Denis C, et al. Early-life establishment of the swine gut microbiome and impact on host phenotypes. Environ Microbiol Rep. 2015;7:554-69.

18. Konstantinov SR, Awati AA, Williams BA, Miller BG, Jones P, Stokes CR, et al. Post-natal development of the porcine microbiota composition and activities. Environ Microbiol. 2006;8:1191-9.

19. Parada AE, Needham DM, Fuhrman JA. Every base matters: assessing small subunit rRNA primers for marine microbiomes with mock communities, time series and global field samples. Environ Microbiol. 2016;18:1403-14.

20. Apprill A, McNally S, Parsons R, Weber L. Minor revision to V4 region SSU rRNA 806R gene primer greatly increases detection of SAR11 bacterioplankton. Aquat Microb Ecol. 2015;75:129-37.

21. Bolyen E, Rideout JR, Dillon MR, Bokulich NA, Abnet C, Al-Ghalith GA, et al. Reproducible, interactive, scalable and extensible microbiome data science using QIIME 2. Nat Biotechnol. 2019;37:852-7.

22. Callahan BJ, McMurdie PJ, Rosen MJ, Han AW, Johnson AJA, Holmes SP. DADA2: high-resolution sample inference from Illumina amplicon data. Nat Methods. 2016;13:581-3.

23. Katoh K, Misawa K, Kuma K, Miyata T. MAFFT: a novel method for rapid multiple sequence alignment based on fast Fourier transform. Nucleic Acids Res. 2002;30:3059-66.

24. Price MN, Dehal PS, Arkin AP. FastTree 2: approximately maximum-likelihood trees for large alignments. PLoS One. 2010;5:e9490.

25. Shannon CE. A mathematical theory of communication. Bell Syst Tech J. 1948;27:379-423.

26. Faith DP. Conservation evaluation and phylogenetic diversity. Biol Conserv. 1992;61:1-10.

27. Lozupone C, Knight R. UniFrac: a new phylogenetic method for comparing microbial communities. Appl Environ Microbiol. 2005;71:8228-35.

28. Lozupone CA, Hamady M, Kelley ST, Knight R. Quantitative and qualitative beta diversity measures lead to different insights into factors that structure microbial communities. Appl Environ Microbiol. 2007;73:1576-85.

29. Bray JR, Curtis JT. An ordination of the upland forest communities of Southern Wisconsin. Ecol Monogr. 1957;27:325-49.

30. Bokulich NA, Kaehler BD, Rideout JR, Dillon M, Bolyen E, Knight R, et al. Optimizing taxonomic classification of marker-gene amplicon sequences with QIIME 2's q2-feature-classifier plugin. Microbiome. 2018;6:90.

31. McDonald D, Price MN, Goodrich J, Nawrocki EP, DeSantis TZ, Probst A, et al. An improved Greengenes taxonomy with explicit ranks for ecological and evolutionary analyses of bacteria and archaea. ISME J. 2012;6:610-8. 
32. Bokulich NA, Dillon MR, Zhang Y, Rideout JR, Bolyen E, Li H, et al. q2-longitudinal: longitudinal and paired-sample analyses of microbiome data. mSystems. 2018;3:e00219-18.

33. R Core Team. R: a language and environment for statistical computing. Vienna, Austria: R Foundation for Statistical Computing.

34. Dixon P. VEGAN, a package of R functions for community ecology. J Veg Sci. 2003;14:92730.

35. Robinson MD, McCarthy DJ, Smyth GK. edgeR: a bioconductor package for differential expression analysis of digital gene expression data. Bioinformatics. 2010;26:139-40.

36. Cortes LCP, LeVeque RM, Funk J, Marsh TL, Mulks MH. Development of the tonsillar microbiome in pigs from newborn through weaning. BMC Microbiol. 2018;18:35.

37. Cortes LCP, LeVeque RM, Funk JA, Marsh TL, Mulks MH. Development of the tonsil microbiome in pigs and effects of stress on the microbiome. Front Vet Sci. 2018;5:220.

38. Kanehisa M, Sato Y, Furumichi M, Morishima K, Tanabe M. New approach for understanding genome variations in KEGG. Nucleic Acids Res. 2019;47:D590-5.

39. Caspi R, Altman T, Billington R, Dreher K, Foerster H, Fulcher CA, et al. The MetaCyc database of metabolic pathways and enzymes and the BioCyc collection of pathway/genome databases. Nucleic Acids Res. 2014;42:D459-71.

40. Cusa E, Obradors N, Baldomà L, Badía J, Aguilar J. Genetic analysis of a chromosomal region containing genes required for assimilation of allantoin nitrogen and linked glyoxylate metabolism in Escherichia coli.J Bacteriol. 1999;181:7479-84.

41. Vogels GD, Van der Drift C. Degradation of purines and pyrimidines by microorganisms. Bacteriol Rev. 1976;40:403-68.

42. Lowe BA, Marsh TL, Isaacs-Cosgrove N, Kirkwood RN, Kiupel M, Mulks MH. Defining the "core microbiome" of the microbial communities in the tonsils of healthy pigs. BMC Microbiol. 2012;12:20.

43. Mann E, Pinior B, Wetzels SU, Metzler-Zebeli BU, Wagner M, Schmitz-Esser S. The metabolically active bacterial microbiome of tonsils and mandibular lymph nodes of slaughter pigs. Front Microbiol. 2015;6:1362.

44. Sandrini S, Alghofaili F, Freestone P, Yesilkaya H. Host stress hormone norepinephrine stimulates pneumococcal growth, biofilm formation and virulence gene expression. BMC Microbiol. 2014;14:180.

45. Colson V, Martin E, Orgeur P, Prunier A. Influence of housing and social changes on growth, behaviour and cortisol in piglets at weaning. Physiol Behav. 2012;107:59-64.

46. Li N, Huang S, Jiang L, Wang W, Li T, Zuo B, et al. Differences in the gut microbiota establishment and metabolome characteristics between low- and normal-birth-weight piglets during early-life. Front Microbiol. 2018;9:1798.

47. Li N, Huang S, Jiang L, Dai Z, Li T, Han D, et al. Characterization of the early life microbiota development and predominant lactobacillus species at distinct gut segments of low- and normal-birth-weight piglets. Front Microbiol. 2019;10:797.

48. Arnold JW, Roach J, Azcarate-Peril MA. Emerging technologies for gut microbiome research. Trends Microbiol. 2016;24:887-901.

49. Quan J, Cai G, Ye J, Yang M, Ding R, Wang X, et al. A global comparison of the microbiome compositions of three gut locations in commercial pigs with extreme feed conversion ratios. Sci Rep. 2018;8:4536.

50. Tan Z, Yang T, Wang Y, Xing K, Zhang F, Zhao X, et al. Metagenomic analysis of cecal microbiome identified microbiota and functional capacities associated with feed efficiency in landrace finishing pigs. Front Microbiol. 2017;8:1546. 
51. Yang H, Huang X, Fang S, He M, Zhao Y, Wu Z, et al. Unraveling the fecal microbiota and metagenomic functional capacity associated with feed efficiency in pigs. Front Microbiol. 2017;8:1555.

52. Mateo CD, Peters DN, Stein HH. Nucleotides in sow colostrum and milk at different stages of lactation. J Anim Sci. 2004;82:1339-42.

53. Drake A, Fraser D, Weary DM. Parent-offspring resource allocation in domestic pigs. Behav Ecol Sociobiol. 2008;62:309-19.

54. Munck A, Guyre PM, Holbrook NJ. Physiological functions of glucocorticoids in stress and their relation to pharmacological actions. Endocr Rev. 1984;5:25-44.

55. Arnone M, Dantzer R. Does frustration induce aggression in pigs? Appl Anim Ethol. 1980;6:351-62.

56. Martínez-Miró S, Tecles F, Ramón M, Escribano D, Hernández F, Madrid J, et al. Causes, consequences and biomarkers of stress in swine: an update. BMC Vet Res. 2016;12:171.

57. Blundell J. Pharmacological approaches to appetite suppression. Trends Pharmacol Sci. 1991;12:147-57.

58. Rossi MA, Stuber GD. Overlapping brain circuits for homeostatic and hedonic feeding. Cell Metab. 2018;27:42-56.

59. Smit AN, Patton DF, Michalik M, Opiol H, Mistlberger RE. Dopaminergic regulation of circadian food anticipatory activity rhythms in the rat. PLoS One. 2013;8:e82381.

60. Eisenhofer G, Kopin IJ, Goldstein DS. Catecholamine metabolism: a contemporary view with implications for physiology and medicine. Pharmacol Rev. 2004;56:331-49. 


\section{SUPPLEMENTARY MATERIALS}

Table S1. Body weights of piglets during the study

\begin{tabular}{|c|c|c|c|c|c|c|c|c|c|c|c|c|c|c|c|c|c|c|}
\hline \multirow{2}{*}{ ID } & \multirow{2}{*}{ Sex } & \multirow{2}{*}{ Uc } & \multirow{2}{*}{ B-O } & \multirow{2}{*}{ MS } & \multirow{2}{*}{ FS } & \multirow{2}{*}{ Wean-age } & \multirow{2}{*}{ BW-class } & \multicolumn{11}{|c|}{ Body-weight (kg) / day of age } \\
\hline & & & & & & & & $\mathbf{0 d}$ & $7 \mathrm{~d}$ & $11 \mathrm{~d}$ & $14 \mathrm{~d}$ & $21 \mathrm{~d}$ & $27 \mathrm{~d}$ & $35 \mathrm{~d}$ & $42 d$ & $49 d$ & $55 d$ & $63 \mathrm{~d}$ \\
\hline R1 & $M$ & 33 & 5 & $A$ & $\mathrm{D}$ & 20 & HBW & 2.1 & 4.4 & 5.0 & 5.6 & 6.6 & 7.6 & 11.4 & 14.1 & 16.4 & 20.5 & 24.5 \\
\hline R2 & $\mathrm{F}$ & 27 & 6 & $A$ & $\mathrm{D}$ & 20 & HBW & 1.8 & 2.9 & 3.5 & 4.3 & 5.1 & 5.9 & 9.1 & 12.6 & 15.4 & 18.4 & 22.5 \\
\hline R3 & $\mathrm{F}$ & & 9 & $A$ & $E$ & 27 & LBW & 1.0 & 2.0 & 2.8 & 3.5 & 5.1 & 6.3 & 6.8 & 9.4 & 11.9 & 14.7 & 17.0 \\
\hline R4 & $\mathrm{F}$ & 25 & 12 & A & $E$ & 27 & LBW & 0.9 & 2.4 & 3.3 & 4.2 & 5.9 & 7.3 & 8.4 & 10.6 & 13.2 & 16.0 & 19.0 \\
\hline B1 & $M$ & 26 & 1 & B & $\mathrm{F}$ & 26 & HBW & 1.7 & 3.4 & 4.7 & 5.8 & 7.9 & 9.8 & 11.0 & 13.7 & 15.2 & 17.2 & 20.5 \\
\hline B2 & $M$ & 40 & 5 & B & $\mathrm{F}$ & 26 & HBW & 1.7 & 3.2 & 4.4 & 5.5 & 7.0 & 8.4 & 8.9 & 11.4 & 12.7 & 14.6 & 17.6 \\
\hline B3 & $\mathrm{F}$ & 37 & 6 & B & B & 26 & LBW & 1.0 & 2.0 & 2.7 & 3.4 & 4.5 & 5.6 & 8.1 & 11.2 & 13.3 & 16.7 & 20.5 \\
\hline B6 & $\mathrm{F}$ & 27 & 12 & B & $B$ & 26 & LBW & 1.0 & 2.2 & 3.2 & 4.0 & 5.0 & 6.2 & 8.5 & 11.6 & 14.7 & 17.8 & 22.7 \\
\hline
\end{tabular}

ID, piglet's identification; Uc, umbilical cord circumference (mm); B-O, birth order; MS, maternal sow (A and B); FS, foster sow (D, E, and F); Wean-age, age at weaning (days); BWClass, birthweight class category; HBW, high birthweight; LBW, low birthweight.

In this study, we classified a pig as heavy $(\mathrm{H})$ or light $(\mathrm{L})$ at the point of sampling basing on the following criteria;

\begin{tabular}{lcc}
\hline & Weight range & Bodyweight class \\
\hline At day 11 & $\leq 3.4$ & $\mathrm{~L}$ \\
& $\geq 3.5$ & $\mathrm{H}$ \\
At day 27 & $\leq 6.9$ & $\mathrm{~L}$ \\
& $\geq 7.0$ & $\mathrm{H}$ \\
At day 63 & $\leq 19.9$ & $\mathrm{~L}$ \\
& $\geq 20.0$ & $\mathrm{H}$ \\
\hline
\end{tabular}

Table S2. Average daily gains of piglets during the study

\begin{tabular}{|c|c|c|c|c|c|c|c|c|c|c|c|c|c|c|c|c|}
\hline \multirow{2}{*}{ ID } & \multirow{2}{*}{ Sex } & \multirow{2}{*}{ Uc } & \multirow{2}{*}{ B-O } & \multirow{2}{*}{ MS } & \multirow{2}{*}{ FS } & \multirow{2}{*}{ Wean-age } & \multicolumn{10}{|c|}{ Average daily gain (ADG) / day of age } \\
\hline & & & & & & & $7 d$ & $11 \mathrm{~d}$ & $14 \mathrm{~d}$ & $21 \mathrm{~d}$ & $27 \mathrm{~d}$ & $35 \mathrm{~d}$ & $42 d$ & $49 d$ & $55 \mathrm{~d}$ & $63 \mathrm{~d}$ \\
\hline R1 & M & 33 & 5 & $A$ & $\mathrm{D}$ & 20 & 0.331 & 0.250 & 0.174 & 0.143 & 0.167 & 0.475 & 0.386 & 0.329 & 0.683 & 0.500 \\
\hline R3 & $\mathrm{F}$ & - & 9 & $A$ & $E$ & 27 & 0.141 & 0.170 & 0.217 & 0.229 & 0.200 & 0.063 & 0.371 & 0.357 & 0.467 & 0.288 \\
\hline R4 & $\mathrm{F}$ & 25 & 12 & $A$ & $E$ & 27 & 0.214 & 0.240 & 0.257 & 0.243 & 0.233 & 0.138 & 0.314 & 0.371 & 0.467 & 0.375 \\
\hline B3 & $F$ & 37 & 6 & B & $B$ & 26 & 0.158 & 0.180 & 0.207 & 0.157 & 0.183 & 0.313 & 0.443 & 0.300 & 0.567 & 0.475 \\
\hline B6 & $\mathrm{F}$ & 27 & 12 & B & B & 26 & 0.207 & 0.230 & 0.251 & 0.143 & 0.200 & 0.288 & 0.443 & 0.443 & 0.517 & 0.613 \\
\hline
\end{tabular}

ID, piglet's identification; Uc, umbilical cord circumference (mm); B-O, birth order; MS, maternal sow (A and B); FS, foster sow (D, E, and F); Wean-age, age at weaning (days). In this study, ADG values were categorised as either low ADG (ldg) or high ADG (hdg) as indicated below.

\begin{tabular}{lcc}
\hline & ADG range & ADG class \\
\hline At day 11 & $\leq 0.19$ & $\mathrm{ldg}$ \\
& $\geq 0.20$ & $\mathrm{hdg}$ \\
At day 27 & $\leq 0.22$ & $\mathrm{Idg}$ \\
& $\geq 0.23$ & $\mathrm{hdg}$ \\
At day 63 & $\leq 0.49$ & $\mathrm{ldg}$ \\
& $\geq 0.50$ & $\mathrm{hdg}$ \\
\hline
\end{tabular}


Table S3. Dietary composition of creep, starter and milk replacer

\begin{tabular}{lc}
\hline Creep feed ingredients & Amount \\
\hline D100 & $1 \mathrm{~kg}$ \\
Probiotic 1 & $10 \mathrm{~g}$ \\
Probiotic 2 & $2.5 \mathrm{~g}$ \\
Vitamin mix 1 & $5 \mathrm{~g}$ \\
Colistin ${ }^{1)}$ & $2.5 \mathrm{~g}$ \\
Minerals & $5 \mathrm{~g}$ \\
\hline Starter feed ingredients & \\
\hline D100 & $1 \mathrm{~kg}$ \\
Probiotic 2 & $1.25 \mathrm{~g}$ \\
Minerals & $1.25 \mathrm{~g}$ \\
Probiotic 1 & $3.75 \mathrm{~g}$ \\
Vitamin mix 1 & $1.25 \mathrm{~g}$ \\
Colistin ${ }^{1)}$ & $1.25 \mathrm{~g}$ \\
\hline D100 Milk replacer (MR) approximate composition & \\
\hline Crude protein & $16.50 \%$ \\
Crude fat & $13.00 \%$ \\
Crude fibre & $1.00 \%$ \\
Crude ash & $4.00 \%$ \\
Ca & $0.30 \%$ \\
P & $0.50 \%$ \\
Lysine & $1.50 \%$ \\
Digestible energy & $4,750 \mathrm{kcal} / \mathrm{kg}$ \\
\hline Digestible crude protein & $14.00 \%$ \\
\hline
\end{tabular}

${ }^{11}$ The Colistin formulation contained colistin sulphate at a concentration of $20 \mathrm{~g} / \mathrm{kg}$ 
Table S4. Weaner feeding schedule

\begin{tabular}{|c|c|c|}
\hline Approx. age (d) & Days post-weaning & Ingredients \\
\hline $28-29$ & $1-2$ & Starter feed \\
\hline \multirow[t]{2}{*}{$30-32$} & $3-5$ & D100 + D200 (1:1) \\
\hline & & Probiotic 1 ( $5 \mathrm{~g} / \mathrm{kg}$ of feed $)$ \\
\hline \multirow[t]{3}{*}{$33-39$} & $6-12$ & D 200 \\
\hline & & Colistin $^{1)}(2.5 \mathrm{~g} / \mathrm{kg}$ of feed $)$ \\
\hline & & Probiotic 1 ( $5 \mathrm{~g} / \mathrm{kg}$ of feed $)$ \\
\hline \multirow[t]{3}{*}{$40-42$} & $13-15$ & D200 + D300 (1:1) \\
\hline & & Probiotic 1 ( $2 \mathrm{~g} / \mathrm{kg}$ of feed $)$ \\
\hline & & Organic acids (2 $\mathrm{g} / \mathrm{kg}$ of feed) \\
\hline \multirow[t]{3}{*}{$43-48$} & $16-21$ & D300 \\
\hline & & Ivermectin ${ }^{2)}(0.333 \mathrm{~g} / \mathrm{kg}$ of feed $)$ \\
\hline & & Probiotic 1 ( $2 \mathrm{~g} / \mathrm{kg}$ of feed) \\
\hline \multirow[t]{4}{*}{$48-51$} & $21-24$ & $\mathrm{D} 300+3 \mathrm{H}(1: 1)$ \\
\hline & & Enrofloxacin (1 g/kg of feed) \\
\hline & & Ivermectin ${ }^{2)}(0.333 \mathrm{~g} / \mathrm{kg}$ of feed $)$ \\
\hline & & Probiotic 1 (2 g/kg of feed) \\
\hline \multirow[t]{2}{*}{$52-70$} & 25 & $3 \mathrm{H}$ \\
\hline & & Probiotic 1 ( $2 \mathrm{~g} / \mathrm{kg}$ of feed) \\
\hline
\end{tabular}

Throughout the study the animals had ad libitum access to the feed rations.

${ }^{1)}$ The Colistin formulation contained colistin sulphate at a concentration of $20 \mathrm{~g} / \mathrm{kg}$

${ }^{2)}$ The Ivermectin formulation contained active Ivermectin at a concentration of $6.12 \mathrm{~g} / \mathrm{kg}$.

Table S5. Additives and their compositions

\begin{tabular}{|c|c|c|c|}
\hline Vitamin mix 1 & & Probiotic 1 & \\
\hline Ingredients & (per kg) & Bacillus spp. & $1.0 \times 10^{9} \mathrm{cfu} / \mathrm{kg}$ \\
\hline Vitamin A & $6,000,000 \mathrm{IU}$ & Lactobacillus spp. & $1.0 \times 10^{9} \mathrm{cfu} / \mathrm{kg}$ \\
\hline Vitamin $D_{3}$ & $600,000 \mathrm{IU}$ & Saccharomyces spp. & $1.0 \times 10^{9} \mathrm{cfu} / \mathrm{kg}$ \\
\hline Vitamin E & $1,000 \mathrm{IU}$ & Probiotic 2 & \\
\hline Vitamin $\mathrm{K}_{3}$ & $500 \mathrm{mg}$ & Clostridium butyricum & $1.0 \times 10^{10} \mathrm{cfu} / \mathrm{kg}$ \\
\hline Vitamin $B_{1}$ & $97 \mathrm{mg}$ & Lactose hydrate & \\
\hline Vitamin $B_{2}$ & $600 \mathrm{mg}$ & Glucose & \\
\hline Vitamin $\mathrm{B}_{6}$ & $400 \mathrm{mg}$ & Organic acids & Per kg \\
\hline Vitamin $\mathrm{B}_{12}$ & $400 \mathrm{ug}$ & Formic acid & $300,000 \mathrm{mg}$ \\
\hline Nicotinic acid & $2,400 \mathrm{mg}$ & Citric acid & $100,000 \mathrm{mg}$ \\
\hline Biotin & $40 \mathrm{mg}$ & Propionic acid & $50,000 \mathrm{mg}$ \\
\hline Choline chloride & $25,000 \mathrm{mg}$ & Malic acid & $50,000 \mathrm{mg}$ \\
\hline Folic acid & $200 \mathrm{mg}$ & Complex amino acids & $>25 \%$ \\
\hline $\mathrm{MgSO}_{4}$ & $4,000 \mathrm{mg}$ & Minerals & \\
\hline Ca-pantothenate & $1,000 \mathrm{mg}$ & $\mathrm{Zn}$ & $2.70 \%$ \\
\hline $\mathrm{FeSO}_{4}$ & $8,000 \mathrm{mg}$ & $\mathrm{Cu}$ & $1.70 \%$ \\
\hline $\mathrm{ZnSO}_{4}$ & $230,000 \mathrm{mg}$ & $\mathrm{Mn}$ & $1.30 \%$ \\
\hline $\mathrm{CuSO}_{4}$ & $1,000 \mathrm{mg}$ & $\mathrm{Fe}$ & $1.30 \%$ \\
\hline $\mathrm{MnSO}_{4}$ & $12,000 \mathrm{mg}$ & $\mathrm{Cr}$ & 160 ppm \\
\hline $\mathrm{CaCO}_{3}$ & $800 \mathrm{mg}$ & & \\
\hline Ca-iodine & $500 \mathrm{mg}$ & & \\
\hline
\end{tabular}



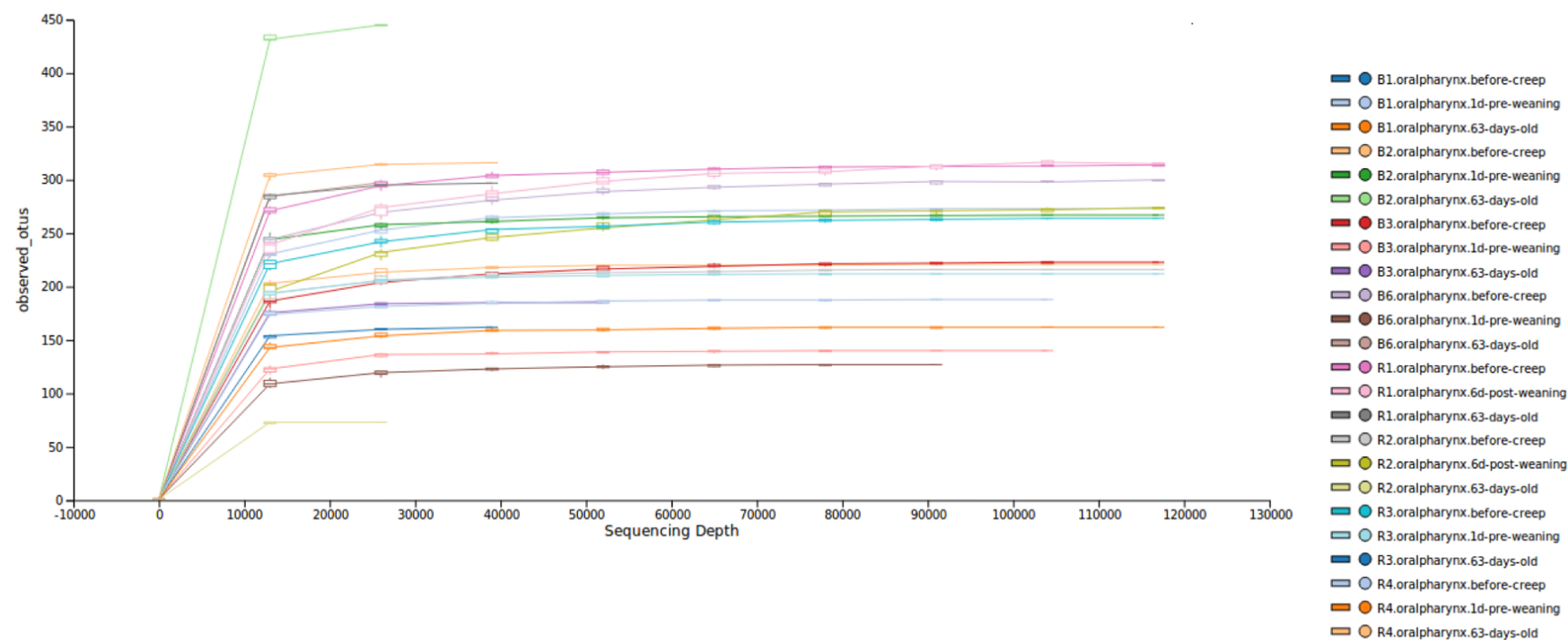

Fig. S1. Alpha rarefaction plot based on observed OTU's showing efficiency of sampling. OTU, operational taxonomic unit.
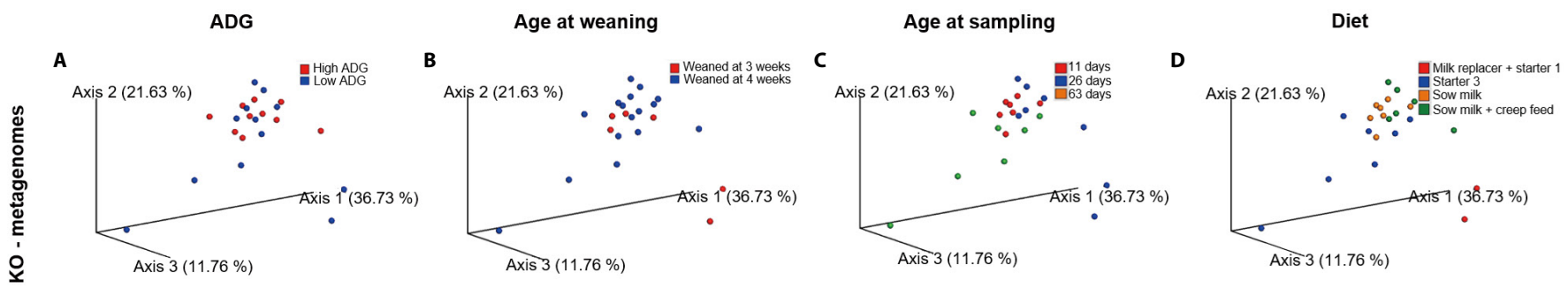

$\mathbf{E}$

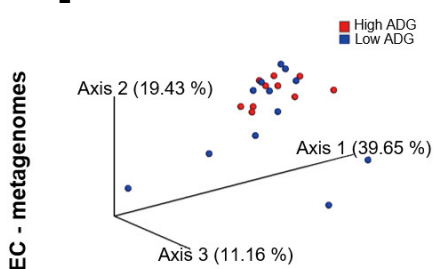

I

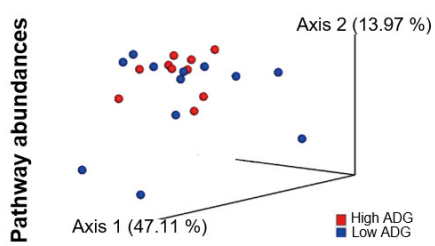

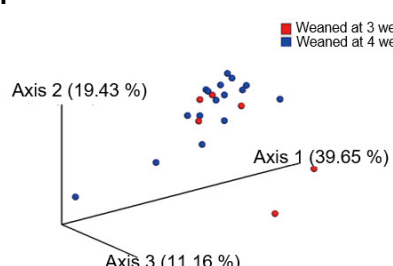

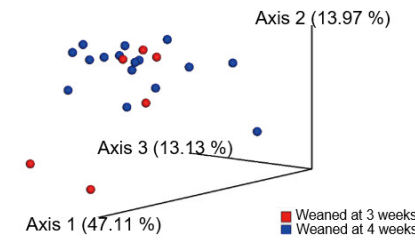

G

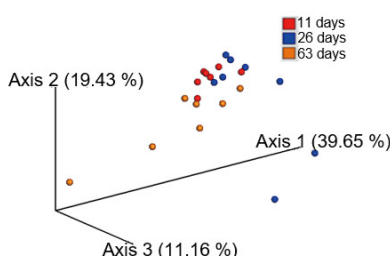

$\mathbf{K}$

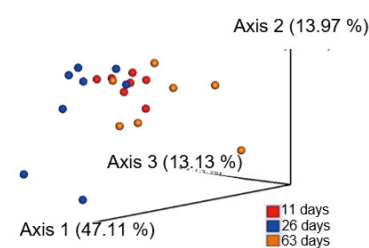

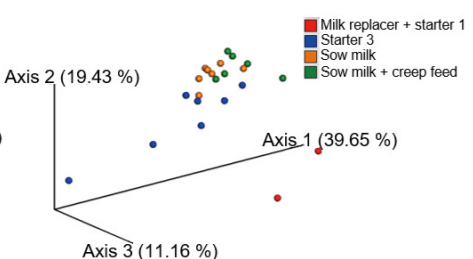

$\mathbf{L}$

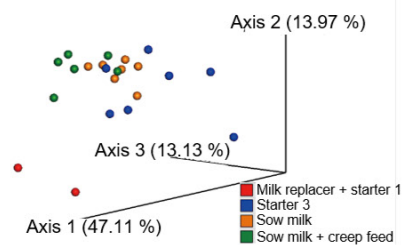

Fig. S2. Principle Coordinate Analysis (PCoA) plots using Bray Curtis distances to show the distribution of oropharyngeal microbiomes in samples collected at 11, 26 and 63 days of age. In (A-D) is the distribution based on the predicted KEGG Orthology (KO) metagenomes; in (E-H), the distribution based on predicted enzymes; and in (I-L) the distribution based on the predicted pathway abundances. The symbols represent data from individual piglets, color-coded by the indicated categories of metadata. 